\title{
Rhythmic and sustained oscillations in metabolism and gene expression of Cyanothece sp. ATCC 51142 under constant light
}

\author{
Sandeep B. Gaudana ${ }^{1 \neq}$, S. Krishnakumar ${ }^{1 \neq}$, Swathi Alagesan ${ }^{1}$, Madhuri G. Digmurti $^{1}$, \\ Ganesh A. Viswanathan ${ }^{1}$, Madhu Chetty ${ }^{2}$ and Pramod P. Wangikar ${ }^{1}$ *
}

${ }^{1}$ Department of Chemical Engineering, Indian Institute of Technology Bombay, Powai, Mumbai, India

${ }^{2}$ Gippsland School of Information Technology, Monash University, VIC, Australia

Edited by:

Thomas E. Hanson, University of

Delaware, USA

\section{Reviewed by:}

Kathleen Scott, University of South

Florida, USA

Ivan Berg, Albert-Ludwigs-Universität

Freiburg, Germany

\section{*Correspondence:}

Pramod P. Wangikar, Department of Chemical Engineering, Indian Institute of Technology Bombay, Powai,

Mumbai 400076, India

e-mail: wangikar@iitb.ac.in

${ }^{\dagger}$ Present address:

Sandeep B. Gaudana, MSU-DOE Plant

Research Laboratory, Michigan State

University, East Lansing, 48824 MI,

USA.

${ }^{\ddagger}$ Sandeep B. Gaudana and

S. Krishnakumar have contributed equally to this work.
Cyanobacteria, a group of photosynthetic prokaryotes, oscillate between day and night time metabolisms with concomitant oscillations in gene expression in response to light/dark cycles (LD). The oscillations in gene expression have been shown to sustain in constant light (LL) with a free running period of $24 \mathrm{~h}$ in a model cyanobacterium Synechococcus elongatus PCC 7942. However, equivalent oscillations in metabolism are not reported under LL in this non-nitrogen fixing cyanobacterium. Here we focus on Cyanothece sp. ATCC 51142, a unicellular, nitrogen-fixing cyanobacterium known to temporally separate the processes of oxygenic photosynthesis and oxygen-sensitive nitrogen fixation. In a recent report, metabolism of Cyanothece 51142 has been shown to oscillate between photosynthetic and respiratory phases under $L L$ with free running periods that are temperature dependent but significantly shorter than the circadian period. Further, the oscillations shift to circadian pattern at moderate cell densities that are concomitant with slower growth rates. Here we take this understanding forward and demonstrate that the ultradian rhythm under LL sustains at much higher cell densities when grown under turbulent regimes that simulate flashing light effect. Our results suggest that the ultradian rhythm in metabolism may be needed to support higher carbon and nitrogen requirements of rapidly growing cells under LL. With a comprehensive Real time PCR based gene expression analysis we account for key regulatory interactions and demonstrate the interplay between clock genes and the genes of key metabolic pathways. Further, we observe that several genes that peak at dusk in Synechococcus peak at dawn in Cyanothece and vice versa. The circadian rhythm of this organism appears to be more robust with peaking of genes in anticipation of the ensuing photosynthetic and respiratory metabolic phases.

Keywords: diazotrophic cyanobacteria, diurnal rhythm, kaiC, nif, RT-PCR

\section{INTRODUCTION}

Cyanobacteria are a group of photosynthetic prokaryotes that inhabit diverse ecosystems. They are believed to have played a role in conversion of the anoxic environment of earth to the present oxic one (Kasting, 2001). Cyanobacterial metabolism oscillates principally between day time metabolism including oxygenic photosynthesis and night time metabolism, of which some reactions may not be compatible with molecular oxygen (Liu et al., 1996; Kondo and Ishiura, 2000). It has now been established that these metabolic rhythms in cyanobacteria are controlled by a circadian clock rather than a real-time sensing of the light availability although the clock does get entrained by the external cues such as light and temperature. Thus, cyanobacteria are emerging as a model system for studies on circadian rhythms (Golden and Canales, 2003). Mechanism of cyanobacterial circadian clock has been experimentally enumerated in a model organism Synechococcus sp. PCC 7942 (henceforth Synechococcus 7942). The core clock comprises of three proteins KaiA, KaiB, and KaiC (Ishiura et al., 1998; Iwasaki et al., 1999). Of these, KaiC is the central oscillator that oscillates between its hyperphosphorylated and hypophosphorylated states at a frequency of $24 \mathrm{~h}$, not just in vivo, but also in vitro when incubated with KaiA, KaiB, and ATP under appropriate conditions (Nakajima etal., 2005). The core clock proteins receive signals from the external environmental cues through the elements of the input pathway such as CikA, which is a histidine kinase and also known as a pseudobacteriophytochrome (Schmitz et al., 2000; Ivleva et al., 2006). As opposed to the initial belief, CikA has been found to sense the redox state rather than acting as a light absorbing photoreceptor (Ivleva et al., 2006). The output pathway is mediated through a sensory histidine kinase, SasA, and a transcription factor RpaA. While promoter activities and gene expression show a circadian rhythm, this does not translate into rhythmic oscillations in metabolism in Syncechococcus 7942. To exemplify, the photosynthesis rates oscillate in tandem with the promoter activity of $p s b A 1$, a key photosystem II gene, only in alternate light/dark (LD) but not in constant light (LL) conditions (Liu et al., 1995; Yen et al., 2004; Johnson et al., 2011). 
In addition to the carbon cycle, cyanobacteria also play an important role in the nitrogen cycle via nitrogenase dependent fixation of atmospheric nitrogen (Sherman et al., 2010; Vijayan and O'Shea, 2013). The nitrogenase enzyme is irreversibly inhibited by oxygen, which necessitates mechanisms for its separation, either spatial or temporal, from the oxygenic photosynthesis in cyanobacteria (Dean et al., 1993). Unicellular nitrogen fixing cyanobacteria such as Cyanothece sp. ATCC 51142 (henceforth Cyanothece 51142) engage in night-time nitrogen fixation concomitantly with respiratory metabolism (Reddy et al., 1993; Schneegurt et al., 1997; Schneegurt et al., 2000; Alagesan et al., 2013; Bandyopadhyay et al., 2013). Respiration not only quenches oxygen but also supplies the energy required for nitrogen fixation by utilizing the glycogen granules stored during the day-time photosynthesis (Schneegurt et al., 1994). Microarray gene expression studies indicate that at least 30 and $10 \%$ of its genes exhibit circadian oscillations under LD (Stöckel et al., 2008) and LL (Toepel et al., 2008) conditions, respectively. In fact, some of the genes show an ultradian rhythm with two cycles of oscillations in a single diurnal period (Elvitigala et al., 2009). Further, culturing in a bioreactor has demonstrated sustained oscillations in optical density (OD) and levels of dissolved carbon dioxide and oxygen under LL (červený and Nedbal, 2009). Recently, červený et al. (2013) have shown ultradian rhythm in Cyanothece 51142 under continuous light. This and the other studies mentioned above, provide an excellent platform for the present study where we show how the oscillations in metabolism and gene expression in this organism are linked to the circadian clock components. We present a comprehensive real time PCR based study with representative genes from key metabolic pathways to demonstrate the interplay among them and their connection with the genes coding for the clock proteins. červený et al. (2013) have demonstrated temperature dependence of ultradian rhythm under continuous light concluding that the ultradian rhythm may be independent of the circadian clock. They also propose that ultradian rhythm is conditional to low culture density and elevated $\mathrm{CO}_{2}$ requirements. In contrast, under our experimental set up of simulated flashing light effect (Krishnakumar et al., 2013), we observe rhythmic ultradian oscillations at gene expression and phenotypic level, which sustain even at higher cell densities and ambient $\mathrm{CO}_{2}$. Further, we observe that the transcription of the core clock genes and genes of the input and output pathway of the circadian clock, which oscillate at a frequency of $24 \mathrm{~h}$ under LD, reset their period to $\sim 11 \mathrm{~h}$ under LL. This encourages us to believe that the ultradian rhythm may in fact be derived from the core circadian clock components and may indeed be the free running period for this organism, as exhibited both at gene expression as well as phenotypic level. We also bring out key differences in gene expression patterns of Cyanothece 51142 and Synechococcus 7942.

\section{MATERIALS AND METHODS CULTURE CULTIVATION AND SAMPLING}

Cyanothece 51142 cultures were grown in an externally illuminated, air sparged and stirred photobioreactor in $1.7 \mathrm{~L} \mathrm{ASP2}$ medium without sodium nitrate (Reddy etal., 1993) at $30^{\circ} \mathrm{C}$ as described earlier (Krishnakumar et al., 2013) with a minor modification. We used an enhanced surface illumination of $230 \mu \mathrm{M}$ photons in the present study. Exhaust gas profiles together with $\mathrm{pH}$ profile have been used to monitor the physiological state of the culture (Bapat et al., 2006; Maiti et al., 2009; Nigam et al., 2012; Krishnakumar et al., 2013). The online and offline parameters of the culture were monitored as described earlier (Krishnakumar et al., 2013). Briefly, the concentrations of carbon dioxide and oxygen in the exit gas from the photobioreactor were analyzed using BlueInOne Cell exit gas analyzer (BlueSens, Herten, Germany). $\mathrm{pH}$ of the growth medium was monitored with a $\mathrm{pH}$ probe (Hamilton, Bonaduz, Switzerland). Cell growth was monitored by measuring the OD of culture at $730 \mathrm{~nm}\left(\mathrm{OD}_{730}\right)$ using a Nanophotometer (Implen, München, Germany). Chlorophyll "a" was extracted by adding $1 \mathrm{ml}$ methanol to cell pellets and heating at $60^{\circ} \mathrm{C}$ for $15 \mathrm{~min}$ and cooled to room temperature. Supernatant was collected after centrifugation at $12000 \mathrm{~g}$ for $15 \mathrm{~min}$ and absorbance was measured spectrophotometrically at 620,678, and $750 \mathrm{~nm}$ (Arnon et al., 1974). Intracellular glycogen from the pellet obtained after chlorophyll extraction was hydrolyzed using 2N HCL (Reddy etal., 1993; Bandyopadhyay et al., 2010) and the released glucose was estimated using Glucose GOD PAP kit, a Glucose oxidase based enzyme assay kit (Biolab Diagnostics (I) Pvt. Ltd., Boisar, India). The culture was entrained under $12 \mathrm{~h}$ LD for $96 \mathrm{~h}$ followed by a free run under LL conditions. Nine samples were drawn from LD and 15 samples from LL for offline measurements such as growth, chlorophyll content, intracellular glycogen concentrations and for gene expression analysis. Samples were drawn from LD condition as described earlier (Krishnakumar et al., 2013). During LL, samples were drawn at times when the culture underwent transitions in its photosynthetic and respiratory phases, as indicated by the exit $\mathrm{CO}_{2}$ and $\mathrm{O}_{2}$ profiles. The data for concentrations of $\mathrm{CO}_{2}$ and $\mathrm{O}_{2}$ in exit gas was fitted to sinusoidal curves using cosine function Eq. 1 described earlier (Kondo et al., 1997) and using the optimize.curve_fit function of the SciPy library for Python.

$$
B=2^{-(t / D)}\{A[\cos (2 \pi t / \mathrm{T}+2 \pi \mathrm{I} / 24)+1]+C\}
$$

Where $B$ is concentration of exit gas $\mathrm{CO}_{2} / \mathrm{O}_{2}$ in volume percent, $T$ is period of circadian/ultradian rhythm, $D$ denotes generation time (h), $A$ denotes amplitude of the rhythmic component, $C$ is a constitutive component, and I is the phase offset of the rhythm on a 24-h scale.

\section{RNA EXTRACTION AND GENE EXPRESSION ANALYSIS}

For extraction of total RNA, samples were drawn at predetermined intervals, centrifuged at $10000 \mathrm{~g}$ for $5 \mathrm{~min}$ at $4^{\circ} \mathrm{C}$ and $1 \mathrm{ml}$ of pre-cooled TRI reagent (Sigma-Aldrich, St Louis, USA) was added and cells were stored at $-80^{\circ} \mathrm{C}$ till further processing. The sample volume was adjusted with the varying culture density to use uniform quantity of biomass for RNA extraction. This was achieved by using sample volume such as the product of volume and $\mathrm{OD}_{730}$ was 10.0. Total RNA was extracted from the samples using TRI reagent (Sigma-Aldrich, St. Louis, MO) as described previously (Krishnakumar et al., 2013) with a minor modification which included the vortexing of thawed 
samples with $500 \mu \mathrm{m}$ diameter acid washed glass beads (SigmaAldrich, St Louis, USA). We selected genes as representatives of photosystem I and II, nitrogenase complex, nitrogen regulation pathway, the clock comprising of kai genes, the input and output pathways of the clock, glycogen synthesis and degradation, carbon fixation, and cell division (Table 1). Gene expression analysis was performed using quantitative Real time PCR (qRT-PCR) analysis using LuminoCt SYBR Green qPCR ReadyMix (SigmaAldrich) on LightCycler ${ }^{\circledR} 480$ (Roche Diagnostics, Indianapolis, IN) and gene specific primers (Table 1) and as described earlier (Gaudana etal., 2013; Krishnakumar etal., 2013). The abundance in expression of individual genes was normalized with that of 16S rRNA gene as endogenous control and the equimolar pool of all RNA samples was used as control for calculating fold changes in expression as described earlier (Stöckel et al., 2008). The qRT-PCR data was clustered by hierarchical centroid linkage algorithm and euclidian distance measure using Cluster 3.0 open source software (de Hoon et al., 2004). The results of the clustering were visualized using a Java Tree view software (Saldanha, 2004).

\section{RESULTS AND DISCUSSION OSCILLATIONS IN METABOLISM}

Under LD conditions, respiratory bursts are observed around the light switch-off time and once every $24 \mathrm{~h}$ (Figures 1A,B and 2). We observe a respiratory burst at approximately $12 \mathrm{~h}$ into constant light $\left(\mathrm{LL}_{12}\right)$, which lasts for about $2 \mathrm{~h}$ followed by a photosynthetic phase. This is also approximately $24 \mathrm{~h}$ after the last respiratory burst in the LD phase. Subsequent respiratory bursts were observed at intervals of $\sim 11 \mathrm{~h}$ with intervening photosynthetic phases. Respiratory bursts are typified by net $\mathrm{CO}_{2}$ evolution, net $\mathrm{O}_{2}$ consumption and a drop in $\mathrm{pH}$ and last for about $2 \mathrm{~h}$. The oscillations sustained for at least $84 \mathrm{~h}$ into LL, the duration for which data was collected (Figure 2). In the present study, we used surface illumination of $230 \mu \mathrm{M}$ photons, an optimal value for the current vessel geometry and hydrodynamic conditions. This is in contrast to earlier reports where surface illumination of 50-90 $\mu \mathrm{M}$ photons has been used (Colón-López and Sherman, 1998; červený and Nedbal, 2009; Krishnakumar et al., 2013). The turbulent regime ensures flashing light effect thereby alleviating potential artifacts arising out of light limitation or light inhibition.

The data for $\mathrm{CO}_{2}$ and $\mathrm{O}_{2}$ concentration in the exhaust gas was fit in sinusoidal curves which denoted a period of 23.26, 10.65, 23.47, and $10.54 \mathrm{~h}$ for $\mathrm{CO}_{2}$ under $\mathrm{LD}, \mathrm{CO}_{2}$ under $\mathrm{LL}, \mathrm{O}_{2}$ under $\mathrm{LD}$ and $\mathrm{O}_{2}$ under LL respectively. The ultradian rhythm between the alternate cycles of carbon and nitrogen fixation in this organism principally seems out of the requirement to meet the demand of high levels of both carbon and nitrogen for rapidly growing cells under LL condition.

Nighttime biomass loss (NBL) is a well known phenomenon in nitrogen fixing unicellular cyanobacteria (Ogbonna and Tanaka, 1996; Carlozzi, 2003). This is usually ascribed to decrease in intracelluar glycogen content (Ogbonna and Tanaka, 1996). We observe NBL not only during LD conditions but also during LL conditions coinciding with the respiratory bursts. The intracellular glycogen content oscillates reaching a peak value just before the respiratory bursts during LL conditions. While NBL coincides with the decrease in glycogen content, the amplitudes of glycogen and biomass oscillations are smaller in LL condition than those in LD conditions (Figure 1C).

\section{OSCILLATIONS IN GENE EXPRESSION}

Stöckel et al. (2008) have reported rhythmic changes in expression of $\sim 30 \%$ of the genes in response to LD cycles. A large number of metabolic genes showed oscillations suggesting prevalence of differences between metabolic activities that occur under the light and the dark periods. The rhythm sustained in some of the genes for the first $24 \mathrm{~h}$ into LL (Toepel et al., 2008). Therefore, it was of interest to see if gene expression shows rhythmic changes under LL beyond the first $24 \mathrm{~h}$ and if these oscillations correlate with those in metabolism (Figure 3). The results, in general, are in agreement with the microarray gene expression results obtained for two diurnal cycles (Stöckel et al., 2008) and for a diurnal cycle followed by constant light for $24 \mathrm{~h}$ (Toepel et al., 2008). Some genes such as $p s a A, r b c L$, cikA, rpaA, nifX, and nifH, show a delayed response in the microarray results (Stöckel etal., 2008; Toepel etal., 2008). These may be either due to the differences in cultivation conditions, the techniques of gene expression studies or sampling frequency or a combination thereof. Interestingly, gene expression pattern of patB is out of phase with that of both the microarray studies. Clustering of our gene expression data (Figure 4) shows that the genes can be broadly categorized as dawn-peaking and duskpeaking (Figures 3A-F and 4) which is consistent with earlier report (Stöckel et al., 2008). Majority of the genes tested under the present study oscillate at a frequency of $24 \mathrm{~h}$ under LD and a frequency of about $11 \mathrm{~h}$ under LL condition with a few exceptions. The genes $f t s Z, c c m k 2$, and $r b c L$ peak twice in a 24$\mathrm{h}$ period, even under LD (Figure 3E). This has been reported earlier from microarray gene expression data for a few other genes (but not for $f t s Z, c c m K 2$, and $r b c L$ genes) and such genes were designated as those with ultradian rhythm (Elvitigala et al., 2009).

\section{OSCILLATIONS IN GENES OF PHOTOSYSTEM I AND II}

The genes $p s a A$ and $p s b A 1$ are generally used as representatives of the photosystem I and II, respectively (Colón-López and Sherman, 1998). In fact, the $p s b A$ promoter has been shown to oscillate in Synechococcus 7942 in constant light with a free run period of $24 \mathrm{~h}$ (Kondo et al., 1993). Further the $p s b A 1$ expression and protein levels have been shown to be up-regulated for about $2 / 3$ rd of the light phase in LD in Cyanothece 51142 (Colón-López and Sherman, 1998). While the $p s a A$ and $p s b A 1$ genes peak (and bottom out) once every $24 \mathrm{~h}$ under LD conditions (Figure 3D and Colón-López and Sherman, 1998), they oscillate at intervals of $\sim 11$ h under LL conditions. The peaks coincide with late respiratory phase thereby preparing the photosynthetic machinery for the ensuing photosynthesis phase. Likewise, $c \mathrm{cmK} 2$, the gene involved in carbon concentrating mechanism, $r b c L$, the gene for large subunit of Rubisco enzyme and ftsZ, a gene involved in cell division, oscillate in tandem with $p s a A$ and $p s b A 1$ under LL condition (Figure 3E). Thus, the entire machinery gets upregulated and ready before the photosynthetic phase. 
Table 1 | List of genes and the respective primer sequences used in the quantitative RT-PCR based gene expression analysis.

\begin{tabular}{|c|c|c|c|}
\hline Gene symbol & Accession Id & Function & Primer sequences \\
\hline \multirow[t]{2}{*}{ cikA } & cce_4751 & Two-component hybrid sensor and regulator & F 5'cacctacgagtcacgggttt \\
\hline & & & R 5'gacaatttgcccttgacgat \\
\hline \multirow[t]{2}{*}{ kaiA } & cce_0424 & Circadian clock protein & F 5'ggaaccctcgtacccgttat \\
\hline & & & R 5'aacccgataacgcacttcag \\
\hline kaiB1 & cce_0423 & Circadian clock protein & F 5'aattttaccccctcctgtcc \\
\hline kaiB2 & & & R $5^{\prime}$ ttttctcgttcggcctgtt \\
\hline \multirow[t]{2}{*}{ kaiB3 } & cce_0435 & Putative circadian clock protein & F $5^{\prime}$ tccatcatctcctcgaaacc \\
\hline & & & R 5' ggtaggcgttgcagaaacat \\
\hline kaiB4 & cce_0145 & Putative circadian clock protein & F $5^{\prime}$ gccatgaaatctacctattccc \\
\hline kaic2 & & & R $5^{\prime}$ ccgttaccatcatcggttct \\
\hline \multirow{2}{*}{ rpaA } & cce_0298 & Two-component response regulator & F 5' cgggctttactcagacgaac \\
\hline & & & R $5^{\prime}$ aaccaaatggcttcaaatcg \\
\hline \multirow[t]{2}{*}{ sas $A$} & cce_1751 & Adaptive-response sensory kinase & F $5^{\prime}$ aaaacaccccttttgcttca \\
\hline & & & $\mathrm{R} 5^{\prime}$ aggtttccttcggcatcttt \\
\hline \multirow[t]{2}{*}{$p s a A$} & cce_0989 & Photosystem I P700 chlorophyll a apoprotein A1 & F 5' tgccaccctatccctaccag \\
\hline & & & R 5'gggctccagcaccaactatt \\
\hline \multirow[t]{2}{*}{$p s b A 1$} & cce_3501 & Photosystem II D1 protein & $F 5^{\prime}$ atctttattctccgctatgc \\
\hline & & & R $5^{\prime}$ tcttgtccgaacttgtaaccg \\
\hline$r b c L$ & cce_3166 & Ribulose bisphosphate carboxylase & F 5' tgccaccctatccctaccag \\
\hline$g \lg P 1$ & & & R 5' caggggttctcaaaccgtaa \\
\hline \multirow[t]{2}{*}{ ccmK2 } & cce_4283 & Carbon dioxide concentrating mechanism protein & F 5' cgtgttacggttattgtgcg \\
\hline & & & R $5^{\prime}$ gtcgatagaacctctcccc \\
\hline \multirow[t]{2}{*}{ nifH } & cce_0559 & Nitrogenase iron protein & F 5'taccattgctgcgttagctg \\
\hline & & & R 5'gtgcagaatggtggtttgtg \\
\hline \multirow[t]{2}{*}{ nifX } & cce_0565 & Nitrogen fixation protein & F 5'gacccccattaaagcgagaa \\
\hline & & & R $5^{\prime}$ ttaaccaaggaggcggattt \\
\hline \multirow[t]{2}{*}{$n t c A$} & cce_0461 & Nitrogen-responsive regulatory protein & F 5'aattttaccccctcctgtcc \\
\hline & & & R $5^{\prime}$ tcgttttcccgttctttgat \\
\hline \multirow[t]{2}{*}{ PatB } & cce_1898 & Cytochrome b6, transcriptional regulator for nitrogen fixation & F 5'agcgatcgccaatctcttt \\
\hline & & & R $5^{\prime}$ tttctcgttcggcctgtt \\
\hline \multirow[t]{2}{*}{$\operatorname{cph} A$} & cce_2237 & Cyanophycin synthetase & $F 5^{\prime}$ tgatcacctggggttaggag \\
\hline & & & R $5^{\prime}$ taagcactgcgtaaccatcg \\
\hline \multirow[t]{2}{*}{ ftsZ } & cce_1314 & Cell division protein & F 5' gatgaacgggttcaaggaga \\
\hline & & & R $5^{\prime}$ ttaggggttgaggtgctttg \\
\hline rrn16Sa & cce_RNA045 & Constitutive expression & F 5' ccctgggctacacacgtact \\
\hline & & & R $5^{\prime}$ tctcgagttgcagagagcaa \\
\hline
\end{tabular}

This includes genes coding for core clock proteins, proteins involved in input and output pathways of circadian clock and select genes coding for proteins involved in key metabolic pathways. 


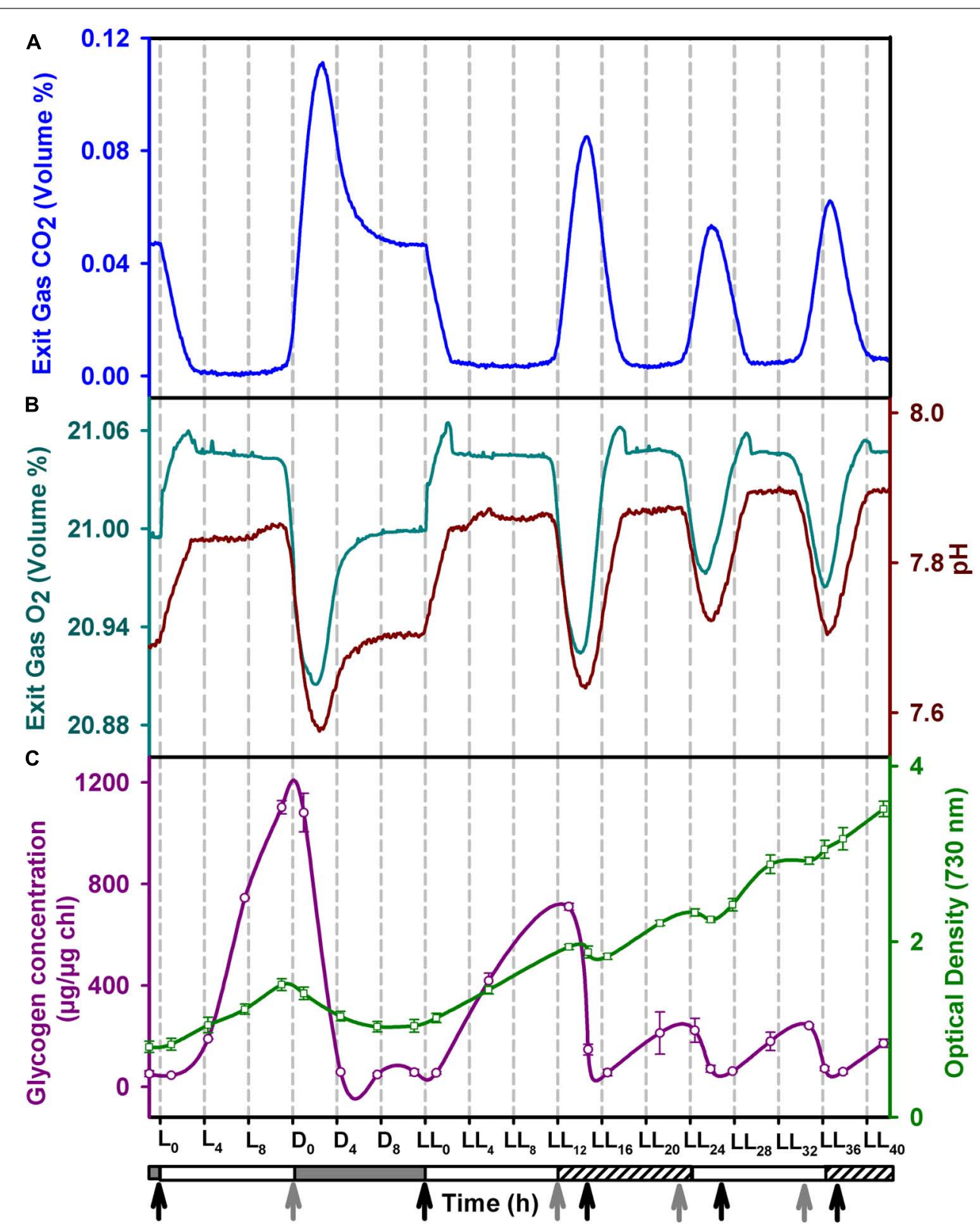

FIGURE 1 | Profiles of (A) $\mathrm{CO}_{2}$ and (B) $\mathrm{O}_{2}$ in the exit gas (cyan), $\mathrm{pH}$ of the growth medium (brown), (C) intracellular glycogen (red) content and growth (green) in the fourth day of entrainment under alternating light/dark cycles (LD) followed by constant light (LL). The horizontal bar below the $X$-axis denotes the light (clear), dark (shaded) and subjective dark (shaded with slanted lines) phases. The onset of photosynthetic and respiratory phases are marked with black and grey arrows, respectively. Error bars on the glycogen and OD data points denote \pm SEM for $N=3$.

\section{OSCILLATIONS IN GENES OF NITROGEN METABOLISM}

The respiratory bursts in LD and LL are preceded by peaks in nifH and nif $X$ genes indicating that the cells gear up for nitrogen fixation which needs to be synchronized with the respiratory burst. Under LD, nif genes peak at time point $\mathrm{L}_{11}$, this is an hour before the onset of dark (Figure 3F). Prior studies have shown peaking of nif genes only after the onset of dark with steady levels of transcript through the night under LD conditions (Stöckel et al., 2008). On the other hand, we observe complete degradation of the nif gene transcripts through the night after the pre-dusk peak.
This suggests that the culturing conditions may have a bearing on the diurnal rhythm of the organism. Under LL, we observe rhythmic oscillations in the nif genes with a period of $\sim 11 \mathrm{~h}$, similar to that for the photosynthesis genes as well as the physiological parameters measured in terms of exit gas and media $\mathrm{pH}$ profiles as well as intracellular glycogen content. The expression profiles of $n t c A$ and $p a t B$, the global regulators for nitrogen fixation (Kolodny et al., 2006; McDermott et al., 2011), are out of phase with that of nif genes (Figure 3F). Indeed, the expression of $n t c A$ has been reported to be out of phase with that of the nifHDK 


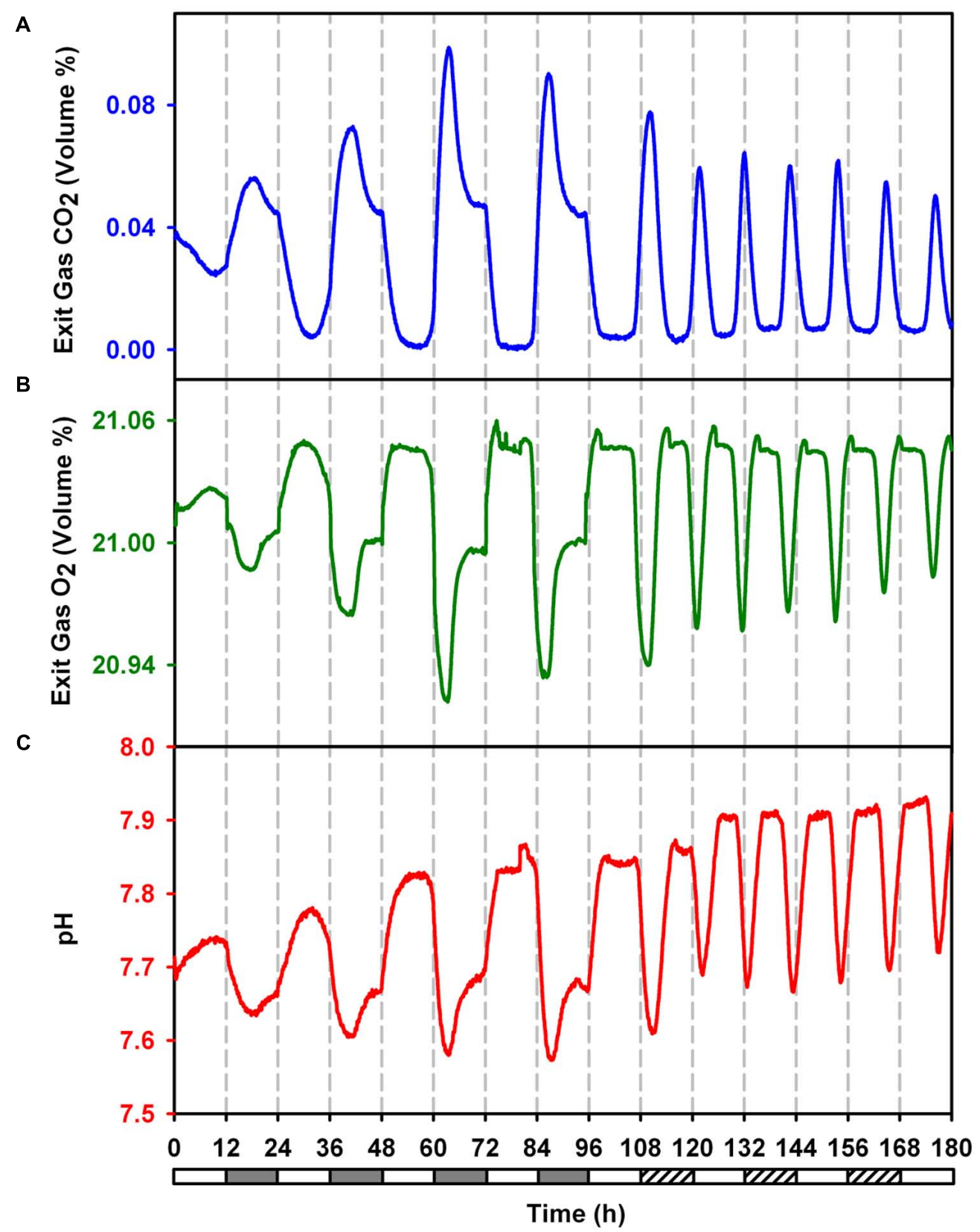

FIGURE 2 | Online monitored exit gas and growth medium pH profiles during 4 days LD followed by 3 days in LL to demonstrate sustained ocillations in metabolism during free run under constant light. (A) Concentration of $\mathrm{CO}_{2}$ in exit gas. (B) Concentration of $\mathrm{O}_{2}$ in exit gas. (C) pH of growth medium.

operon under nitrogen fixing conditions for a Cyanothece strain (Bradley and Reddy, 1997).

\section{OSCILLATIONS IN GENES OF CIRCADIAN RHYTHM}

Molecular mechanism for the circadian clock and its input and output pathway has been extensively reported for Synechococcus 7942. The redox state of intracellular quinone pool is a key factor in the signaling of switch from daytime to nighttime activities (Kim et al., 2012). The intracellular plastoquinone (PQ) pool stays in a reduced state during the light phase with a sudden drop in the reduced pool at the onset of dark (Kim et al., 2012). Only the oxidized form of quinone binds to CikA as well as KaiA and induces the degradation of CikA and aggregation of KaiA (Ivleva et al., 2006; Kim et al., 2012). CikA then induces rpaA and RpaA then dislocates RpaB from the $k a i B C$ operon leading to its induction (Hanaoka et al., 2012).

While homologs of many of the clock genes are present, the regulatory interactions have not been enumerated in other cyanobacteria including Cyanothece 51142. There are some notable differences in the gene expression patterns of Synechococcus 7942 


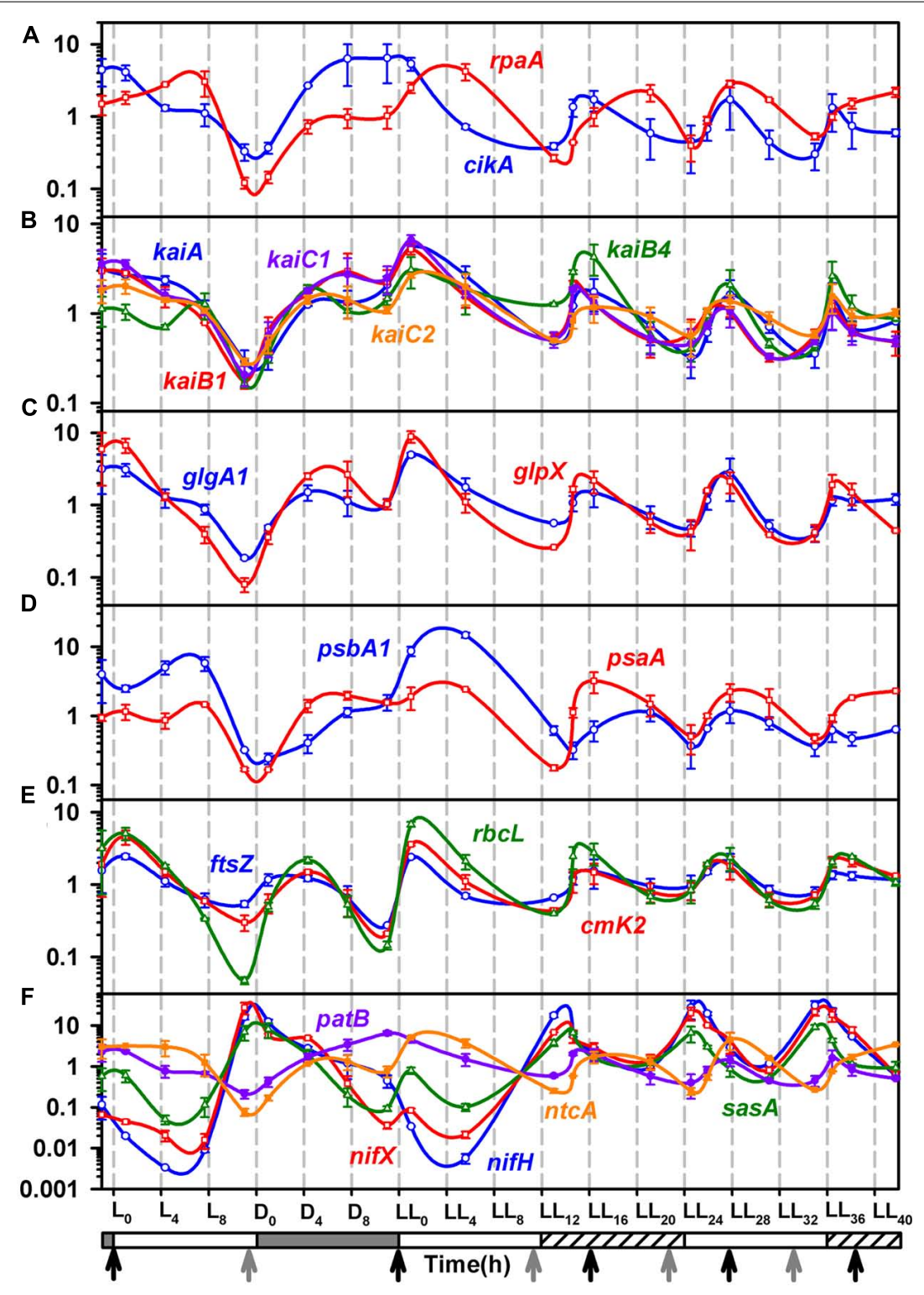

FIGURE 3 | Quantitative Real-time PCR analysis for total RNA samples from the 4th day of entrainment under alternating light/dark cycles (LD) followed by constant light (LL). (A) The level of transcripts for cikA (blue), and rpa (red), involved in input and output pathway and output pathway of circadian rhythm; (B) The trasncription profiles for clock genes, kaiA (blue) kaiB1 (red), kaiB4 (green), kaiC1 (violet) and kaiC2 (orange); (C) The expression profiles of glycogen synthesis genes, glpX (red) and glgA1 (blue) matches closely with that of kai genes, indicating a possible close association of glycogen synthesis with the clock. (D) The transcript profiles of $p s b A 1$ (blue) and psaA (red), genes of Photosystem I and II respectively; (E) The epxpression profiles of genes involved in carbon fixation, $r b c L$ (green) and ccmK2 (red) and cell division, ftsZ (blue); (F) Transcription profiles of the circadian rhythm output pathway gene sas $A$ (green) and genes of nitrogenase complex, nifH (blue) and nifX (red), nitrogen fixation regulation genes pat $B$ (violet) and $n t c A$ (orange). Refer to Figure $\mathbf{1}$ for other details and Table $\mathbf{1}$ for details of genes and PCR primers.
(Ito et al., 2009) and Cyanothece 51142. The genes of the circadian clock and the input and output pathway oscillate at a frequency of $\sim 11 \mathrm{~h}$ in Cyanothece 51142 as compared to that of $24 \mathrm{~h}$ in Synechococcus 7942 under constant light condition. Further, the genes for KaiA, KaiB, KaiC, and CikA homologs in Cyanothece 51142 peak at dawn (Figures 3A,B) in contrast to their peaking at dusk in Synechococcus 7942 (Kondo and Ishiura, 2000; Vijayan and O'Shea, 2013). While the gene regulatory interactions may have been conserved between Synechococcus 7942 and Cyanothece
51142, we propose that the phase differences may originate from the redox state of the PQ pool mediated by glycogen metabolism. We propose that in Cyanothece 51142, the reduced quinone pool declines gradually during the day as a result of glycogen synthesis with a burst in the reduced pool triggered by the respiratory burst at dusk (Figure 5). This might be one of the mechanisms responsible for oxidation of the intracellular quinone pool during the light phase. This is in agreement with the prevailing understanding about the intracellular storage molecules such as 


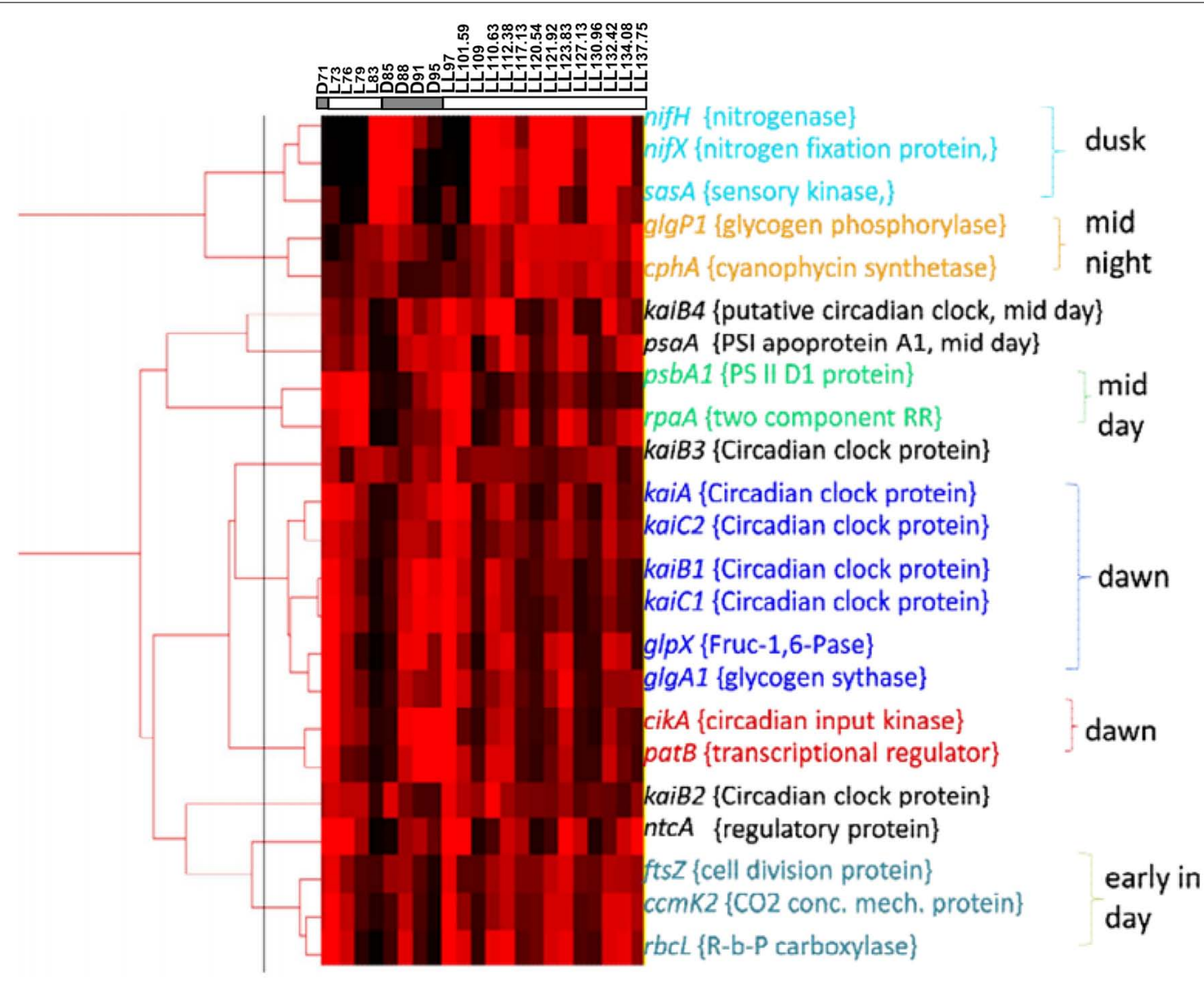

FIGURE 4 | Clustering analysis on basis of the Real time PCR expression data of genes coding for clock genes, input and output pathway of circadian rhythm and selected representatives from genes involved in various metabolic pathways.

glycogen being instrumental in influencing the redox state of the intracellular quinone pool (Kreysa and Krämer, 1989; Sherman et al., 2010). Previously it is shown that glycogen is a key respiratory substrate and that an impaired glycogen synthesis negatively influences metabolic regulations in another cyanobacterium Synechocystis sp. PCC 6803 (Gründel et al., 2012). A direct interaction of glycogen synthase with thioredoxin in Synechocystis 6803 was also recently proposed (Díaz-Troya et al., 2013). Further, we believe that the role of glycogen could be analogous to that played by starch in Arabidopsis, in which the intracellular concentrations of solutes such as glucose and sucrose play a central role in providing a feedback to the circadian clock genes, though the mechanism for this feedback is not understood (Haydon et al., 2011). Thus, glycogen synthesis and degradation processes in Cyanothece 51142 are likely to play a key role in regulating the circadian rhythm on two counts: by dictating the concentration of intracellular glucose and by acting as respiratory substrate. However, further direct experimental evidences are required to validate this hypothesis.

In Cyanothece 51142, there is no noticeable phase lag between the expression of rpaA and the kaiAB1C1 cluster (Figures 3A,B), suggesting that the signal from CikA may be conferred to the kaiAB1C1 cluster by an alternate intermediate other than RpaA. The initiation of transcription of the kaiAB1C1 cluster then leads to the transcription of genes involved in daytime activity such as photosynthesis ( $p s b a A, p s b A 1)$ and glycogen synthesis $(g l p X, g l g A 1$; Figures 3C,D and 5). The gradual oxidization of the quinone pool during the photosynthesis phase, as result of the glycogen synthesis, might be the signal conferred to the nif genes through SasA (Figures 3F and 5).

During the first $2 \mathrm{~h}$ into the first subjective dark phase under LL, the chronology of events remains similar to that as the first $2 \mathrm{~h}$ of the dark phase under LD. The intracellular glycogen concentration falls and so does the exit gas $\mathrm{O}_{2}$ and the $\mathrm{pH}$. However, probably because of the presence of light even during the subjective dark might have caused an early reduction of the quinone pool, since the redox state of the quinone pool is understood to be controlled by the photosynthetic electron transport as a function of light availability and by the respiratory electrons in dark (Kim et al., 2012). This could in turn have shifted the rhythm into the day time activity such as photosynthesis and glycogen synthesis. The same is indicated by the increase in the intracellular 


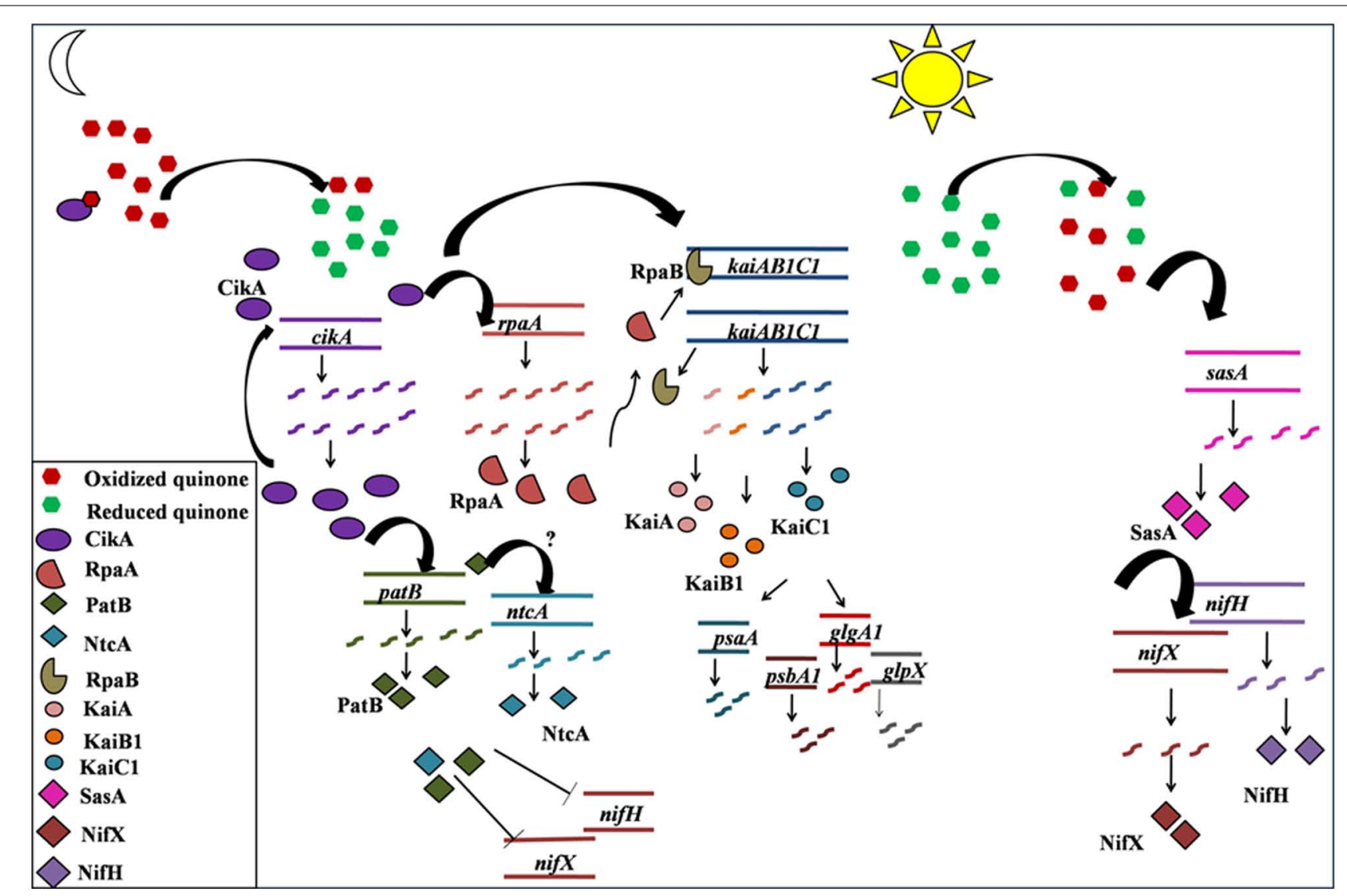

FIGURE 5 | A schematic representation of the proposed sequence of transcription events for temporal separation in Cyanothece sp. ATCC 51142. The predictions are based upon the transcription profiles of genes monitored using RT-PCR in the present study and on the existing understanding of the functioning of different components of circadian rhythm in Synechococcus sp. PCC 7942. Reduction of PQ pool, following the respiratory burst at dusk, leads to activation of CikA (Kim et al., 2012), which inturn activates its own transcription as well as the transcription of
kaiAB1C1cluster, possibly via RpaA as reported for Synechococcus 7942 (Hanaoka etal., 2012) or by an alternate pathway. The transcription and translation of kaiAB1C1 cluster in turn leads to initiation of transcription of genes for photosystem I and II as well as for glycogen synthesis, all of which peak at dawn in anticipation of the ensuing light phase. Oxidation of $\mathrm{PO}$ pool possibly activates transcription of sasA. SasA activates the transcrition of nif genes which peak at dusk and prepare the system for carrying out nitrogen fixation during the respiratory burst. glycogen concentration in the middle of the first subjective dark phase unlike that under the dark phase of LD, where glycogen concentration remains at the base till the initiation of the light phase. This is also synchronous with the upregulation of the phostosynthesis genes $p s b A 1$ and $p s a A$, glycogen synthesis genes $g \lg P 1$ and $g \lg X$ and the kaiA, kaiB1, kaiC1, and kaiC2 gene cluster, which all peak at the $4-6 \mathrm{~h}$ of the first subjective dark and then at a frequency of $11 \mathrm{~h}$ then onward. The beginning of the first subjective dark leads to the peaking of the nif genes and that of intracellular glycogen concentration. These peaks are then observed at a frequency of about $11 \mathrm{~h}$ and in tandem with the above described day time activities such as photosynthesis and glycogen synthesis.

\section{COMPARISON OF CIRCADIAN RHYTHM OF Cyanothece 51142 AND Synechococcus 7942}

Synechococcus 7942 exhibits a free run period of $24 \mathrm{~h}$ with its doubling time of $6 \mathrm{~h}$ (Mori et al., 1996) while Cyanothece shows a free run period of $\sim 11 \mathrm{~h}$ and a doubling time of $30 \mathrm{~h}$ in constant light under the present culturing conditions. This shows that there may not be a correlation between the doubling time and free run period in cyanobacteria. Further, there are some noticeable differences between the two organisms in the terms of the genes of the clock and the output pathway. Specifically, the kaiAB1C1 operon found in Cyanothece 51142 (Memon et al., 2013) is shorter by one gene and present as kaiBC in Synechococcus 7942 (Kutsuna et al., 2005). While Synechococcus 7942 contains only one gene each for KaiA, KaiB, and KaiC, Cyanothece 51142 contains multiple homologs of KaiB and KaiC (Table 1). Importantly, homolog of the period extender protein Pex (Kutsuna et al., 1998; Tanaka et al., 2012) is missing in Cyanothece 51142. However, lack of the pex gene may only partially explain differences in the free run periods of the two organisms. Further, the photosystem genes such as $p s b A 1$ and $p s a A$ peak at dawn and stay upregulated for about 2/3rd of the day in Cyanothece 51142 (Figure 3D) in contrast to the peaking at dusk in Synechococcus 7942 (Kondo and Ishiura, 2000; Vijayan and O'Shea, 2013). Likewise, the nifH and nifX genes peak pre-dusk in anticipation of the ensuing respiratory 
burst (Figure 2F), which is necessary for the nitrogen fixation activity. On the whole, the main contrast between the circadian rhythm of Synechococcus 7942 and Cyanothece 51142 seems to be based upon the robustness that enables Cyanothece 51142 to be prepared for the next metabolic phase during the existing metabolic cycle while it temporally regulates the separation of oxygenic photosynthesis from oxygen sensitive nitrogen fixation process.

The present study, which was conducted under prolonged constant light, provides understanding about the physiological and molecular behavior of Cyanothece 51142 under constant light and is a concrete evidence of a circadian rhythm in and temporal separation of key metabolic processes under this free running condition.

\section{ACKNOWLEDGMENTS}

The work was partially funded by an Australia-India strategic research (AISRF) grant to Pramod P. Wangikar and Madhu Chetty. The grant to the Indian side was provided by Department of Biotechnology, Ministry of Science and Technology, Government of India, grant number: BT/Indo-Aus/04/04/2009. The authors have no conflict of interest to declare.

\section{AUTHOR CONTRIBUTIONS}

Sandeep B. Gaudana and S. Krishnakumar have contributed equally to this work. Sandeep B. Gaudana, S. Krishnakumar and Pramod P. Wangikar designed research; Sandeep B. Gaudana, S. Krishnakumar, Swathi Alagesan and Madhuri G. Digmurti performed research; Sandeep B. Gaudana, S. Krishnakumar, Madhuri G. Digmurti, Ganesh A. Viswanathan, Madhu Chetty and Pramod P. Wangikar analyzed the data; Sandeep B. Gaudana and Pramod P. Wangikar wrote the paper.

\section{REFERENCES}

Alagesan, S., Gaudana, S. B., Sinha, A., and Wangikar, P. P. (2013). Metabolic flux analysis of Cyanothece sp. ATCC 51142 under mixotrophic conditions. Photosynth. Res. 118, 191-198. doi: 10.1007/s11120-013-9911-5

Arnon, D. I., McSwain, B. D., Tsujimoto, H. Y., and Wada, K. (1974). Photochemical activity and components of membrane preparations from blue green algae. Coexistence of two photosystems in relation to chlorophyll a and removal of phycocyanin. Biochim. Biophys. Acta. 357, 231-245. doi: 10.1016/0005-2728(74)90063-2

Bandyopadhyay, A., Elvitigala, T., Liberton, M., and Pakrasi, H. B. (2013). Variations in the rhythms of respiration and nitrogen fixation in members of the unicellular diazotrophic cyanobacterial genus Cyanothece. Plant. Physiol. 161, 1334-1346. doi: 10.1104/pp.112.208231

Bandyopadhyay, A., Stöckel, J., Min, H., Sherman, L. A., and Pakrasi, H. B. (2010). High rates of photobiological $\mathrm{H} 2$ production by a cyanobacterium under aerobic conditions. Nat. Commun. 1, 139. doi: 10.1038/ncomms1139

Bapat, P. M., Das, D., Dave, N. N., and Wangikar, P. P. (2006). Phase shifts in the stoichiometry of rifamycin B fermentation and correlation with the trends in the parameters measured online. J. Biotechnol. 127, 115-128. doi: 10.1016/j.jbiotec.2006.06.014

Bradley, R. L., and Reddy, K. J. (1997). Cloning, sequencing, and regulation of the global nitrogen regulator gene ntcA in the unicellular diazotrophic cyanobacterium Cyanothece sp. strain BH68K. J. Bacteriol. 179, 4407-4410.

Carlozzi, P. (2003). Dilution of solar radiation through "culture" lamination in photobioreactor rows facing south-north: a way to improve the efficiency of light utilization by cyanobacteria (Arthrospira platensis). Biotechnol. Bioeng. 81, 305-315. doi: 10.1002/bit.10478 červený, J., and Nedbal, L. (2009). Metabolic rhythms of the cyanobacterium Cyanothece sp. ATCC 51142 correlate with modeled dynamics of circadian clock. J. Biol. Rhythms 24, 295-303. doi: 10.1177/0748730409338367

červený, J., Sinetova, M. A., Valledor, L., Sherman, L. A., and Nedbal, L. (2013). Ultradian metabolic rhythm in the diazotrophic cyanobacterium Cyanothece sp. ATCC 51142. Proc. Natl. Acad. Sci. U.S.A. 110, 13210-13215. doi: $10.1073 /$ pnas. 1301171110

Colón-López, M. S., and Sherman, L. A. (1998). Transcriptional and translational regulation of photosystem I and II genes in light-dark- and continuous-lightgrown cultures of the unicellular cyanobacterium Cyanothece sp. strain ATCC 51142. J. Bacteriol. 180, 519-526.

Dean, D. R., Bolin, J. T., and Zheng, L. (1993). Nitrogenase metalloclusters: structures, organization, and synthesis. J. Bacteriol. 175, 6737-6744.

de Hoon, M. J., Imoto, S., Nolan J., and Miyano S. (2004). Open source clustering software. Bioinformatics 20, 1453-1454. doi: 10.1093/bioinformatics/bth078

Díaz-Troya, S., López-Maury, L., Sánchez-Riego, A. M., Roldan, M., and Florencio, F. J. (2013). Redox regulation of glycogen biosynthesis in the cyanobacterium Synechocystis sp. PCC 6803. Analysis of the AGP and glycogen synthases. Mol. Plant. doi: 10.1093/mp/sst137 [Epub ahead of print].

Elvitigala, T., Stöckel, J., Ghosh, B. K., and Pakrasi, H. B. (2009). Effect of continuous light on diurnal rhythms in Cyanothece sp. ATCC 51142. BMC Genomics 10:226. doi: 10.1186/1471-2164-10-226

Gaudana, S. B., Alagesan, S., Chetty, M., and Wangikar, P. P. (2013). Diurnal rhythm of an unicellular diazotrophic cyanobacterium under mixotrophic conditions and elevated carbon dioxide. Photosynth. Res. 118, 51-57. doi: 10.1007/s11120-0139888-0

Golden, S. S., and Canales, S. R. (2003). Cyanobacterial circadian clocks - timing is everything. Nat. Rev. Microbiol. 1, 191-199. doi: 10.1038/nrmicro774

Gründel, M., Scheunemann, R., Lockau W., and Zilliges Y. (2012). Impaired glycogen synthesis causes metabolic overflow reactions and affects stress responses in the cyanobacterium Synechocystis sp. PCC 6803. Microbiology 158, 3032-3043. doi: 10.1099/mic.0.062950-0

Hanaoka, M., Takai, N., Hosokawa, N., Fujiwara, M., Akimoto, Y., Kobori, N., et al. (2012). RpaB, another response regulator operating circadian clock-dependent transcriptional regulation in Synechococcus elongatus PCC 7942. J. Biol. Chem. 287, 26321-26327. doi: 10.1074/jbc.M111.338251

Haydon, M. J., Bell, L. J., and Webb A. A. R. (2011). Interactions between plant circadian clocks and solute transport. J. Exp. Bot. 62, 2333-2348. doi: 10.1093/jxb/err040

Ishiura, M., Kutsuna, S., Aoki, S., Iwasaki, H., Andersson, C. R., Tanabe, A., et al. (1998). Expression of a gene cluster kaiABC as a circadian feedback process in cyanobacteria. Science 281, 1519-1523. doi: 10.1126/science.281.5382. 1519

Ito, H., Mutsuda, M., Murayama, Y., Tomita, J., Hosokawa, N., Terauchi, K., et al. (2009). Cyanobacterial daily life with Kai-based circadian and diurnal genomewide transcriptional control in Synechococcus elongatus. Proc. Natl. Acad. Sci. U.S.A. 106, 14168-14173. doi: 10.1073/pnas.0902587106

Ivleva, N. B., Gao, T., Liwang, A. C., and Golden, S. S. (2006). Quinone sensing by the circadian input kinase of the cyanobacterial circadian clock. Proc. Natl. Acad. Sci. U.S.A. 103, 17468-17473. doi: 10.1073/pnas.0606639103

Iwasaki, H., Taniguchi, Y., Ishiura, M., and Kondo, T. (1999). Physical interactions among circadian clock proteins KaiA, KaiB and KaiC in cyanobacteria. EMBO J. 18, 1137-1145. doi: 10.1093/emboj/18.5.1137

Johnson, C. H., Stewart, P. L., and Egli, M. (2011). The cyanobacterial circadian system: from biophysics to bioevolution. Annu. Rev. Biophys. 40, 143-167. doi: 10.1146/annurev-biophys-042910-155317

Kasting, J. F. (2001). The rise of atmospheric oxygen. Science 293, 819-820. doi: 10.1126/science.1063811

Kim, Y.-I., Vinyard, D. J., Ananyev, G. M., Dismukes, G. C., and Golden, S. S. (2012). Oxidized quinones signal onset of darkness directly to the cyanobacterial circadian oscillator. Proc. Natl. Acad. Sci. U.S.A. 109, 17765-17769. doi: 10.1073/pnas.1216401109

Kolodny, N. H., Bauer, D., Bryce, K., Klucevsek, K., Lane, A., Medeiros, L., et al. (2006). Effect of Nitrogen Source on Cyanophycin Synthesis in Synechocystis sp. Strain PCC 6308. J. Bacteriol. 188, 934-940. doi: 10.1128/JB.188.3.934-940.2006

Kondo, T., and Ishiura, M. (2000). The circadian clock of cyanobacteria. BioEssays 22, 10-15. doi: 10.1002/(SICI)1521-1878(200001)22:1<10::AIDBIES4>3.0.CO;2-A 
Kondo, T., Mori, T., Lebedeva, N. V., Aoki, S., Ishiura, M., and Golden, S. S. (1997). Circadian rhythms in rapidly dividing cyanobacteria. Science 275, 224-227. doi: 10.1126/science.275.5297.224

Kondo, T., Strayer, C. A., Kulkarni, R. D., Taylor, W., Ishiura, M., Golden, S. S., et al. (1993). Circadian rhythms in prokaryotes: luciferase as a reporter of circadian gene expression in cyanobacteria. Proc. Natl. Acad. Sci. U.S.A. 90, 5672-5676. doi: 10.1073/pnas.90.12.5672

Kreysa, G., and Krämer, P. (1989). Macrokinetics and mathematical modelling of quinone reduction by cyanobacteria. J. Chem. Technol. Biotechnol. 44, 205-217. doi: $10.1002 /$ jctb. 280440305

Krishnakumar, S., Gaudana, S. B., Viswanathan, G. A., Pakrashi, H. B., and Wangikar, P. P. (2013). Rhythm of nitrogen fixation by Cyanothece sp. ATCC 51142 under fully aerobic conditions. Biotechnol. Bioeng. 2371-2379. doi: 10.1002/bit.24882

Kutsuna, S., Kondo, T., Aoki, S., and Ishiura, M. (1998). A period-extender gene, pex, that extends the period of the Circadian clock in the Cyanobacterium Synechococcus sp. Strain PCC 7942. J. Bacteriol. 180, 2167-2174.

Kutsuna, S., Nakahira, Y., Katayama, M., Ishiura, M., and Kondo, T. (2005). Transcriptional regulation of the circadian clock operon kaiBC by upstream regions in cyanobacteria. Mol. Microbiol. 57, 1474-1484. doi: 10.1111/j.13652958.2005.04781.x

Liu, Y., Tsinoremas, N. F., Golden, S. S., Kondo, T., and Johnson, C. H. (1996). Circadian expression of genes involved in the purine biosynthetic pathway of the cyanobacterium Synechococcus sp. strain PCC 7942. Mol. Microbiol. 20, 10711081. doi: 10.1111/j.1365-2958.1996.tb02547.x

Liu, Y., Tsinoremas, N. F., Johnson, C. H., Lebedeva, N. V., Golden, S. S., Ishiura, M., et al. (1995). Circadian orchestration of gene expression in cyanobacteria. Genes Dev. 9, 1469-1478. doi: 10.1101/gad.9.12.1469

Maiti, S. K., Srivastava, R. K., Bhushan, M., and Wangikar, P. P. (2009). Real time phase detection based online monitoring of batch fermentation processes. Process Biochem. 44, 799-811. doi: 10.1016/j.procbio.2009.03.008

McDermott, J. E., Oehmen, C. S., Mccue, L. A., Hill, E., Choi, D. M., Stöckel, J., et al. (2011). A model of cyclic transcriptomic behavior in the cyanobacterium Cyanothece sp. ATCC 51142. Mol. BioSyst. 7, 2407-2418. doi: $10.1039 / \mathrm{clmb} 05006 \mathrm{k}$

Memon, D., Singh, A. K., Pakrasi, H. B., and Wangikar, P. P. (2013). A global analysis of adaptive evolution of operons in cyanobacteria. Antonie Leeuwenhoek 103, 331-346. doi: 10.1007/s10482-012-9813-0

Mori, T., Binder, B., and Johnson, C. H. (1996). Circadian gating of cell division in cyanobacteria growing with average doubling times of less than 24 hours. Proc. Natl. Acad. Sci. U.S.A. 93, 10183-10188. doi: 10.1073/pnas.93.19.10183

Nakajima, M., Imai, K., Ito, H., Nishiwaki, T., Murayama, Y., Iwasaki, H., et al. (2005). Reconstitution of circadian oscillation of cyanobacterial KaiC phosphorylation in vitro. Science 308, 414-415. doi: 10.1126/science.1108451

Nigam, A., Phale, P. S., and Wangikar, P. P. (2012). Assessment of the metabolic capacity and adaptability of aromatic hydrocarbon degrading strain Pseudomonas putida CSV86 in aerobic chemostat culture. Bioresour. Technol. 114, 484-491. doi: 10.1016/j.biortech.2012.03.007

Ogbonna, J. C., and Tanaka, H. (1996). Night biomass loss and changes in biochemical composition of cells during light/dark cyclic culture of Chlorella pyrenoidosa. J. Ferment. Bioeng. 82, 558-564. doi: 10.1016/S0922-338X(97)81252-4

Reddy, K. J., Haskell, J. B., Sherman, D. M., and Sherman, L. A. (1993). Unicellular, aerobic nitrogen-fixing cyanobacteria of the genus Cyanothece. J. Bacteriol. 175, 1284-1292.

Saldanha A. J. (2004). Java Treeview - extensible visualization of microarray data. Bioinformatics 20 , 3248-3248. doi: 10.1093/bioinformatics/bth349
Schmitz, O., Katayama, M., Williams, S. B., Kondo, T., and Golden, S. S. (2000). CikA, a bacteriophytochrome that resets the cyanobacterial circadian clock. Science 289, 765-768. doi: 10.1126/science.289.5480.765

Schneegurt, M. A., Sherman, D. M., Nayar, S., and Sherman, L. A. (1994). Oscillating behavior of carbohydrate granule formation and dinitrogen fixation in the cyanobacterium Cyanothece sp. strain ATCC 51142. J. Bacteriol. 176, 15861597.

Schneegurt, M. A., Sherman, D. M., and Sherman, L. A. (1997). Composition of the carbohydrate granules of the cyanobacterium, Cyanothece sp. strain ATCC 51142. Arch. Microbiol. 167, 89-98. doi: 10.1007/s002030050420

Schneegurt, M. A., Tucker, D. L., Ondr, J. K., Sherman, D. M., and Sherman, L. A. (2000). Metabolic rhythms of a diazotrophic cyanobacterium, Cyanothece sp. strain ATCC 51142, heterotrophically grown in continuous dark. J. Phycol. 36, 107-117. doi: 10.1046/j.1529-8817.2000.99152.x

Sherman, L. A., Min, H., Toepel, J., and Pakrasi, H. B. (2010). Better living through Cyanothece - unicellular diazotrophic cyanobacteria with highly versatile metabolic systems. Adv. Exp. Med. Biol. 675, 275-290. doi: 10.1007/978-1-44191528-3_16

Stöckel, J., Welsh, E. A., Liberton, M., Kunnvakkam, R., Aurora, R., and Pakrasi, H. B. (2008). Global transcriptomic analysis of Cyanothece 51142 reveals robust diurnal oscillation of central metabolic processes. Proc. Natl. Acad. Sci. U.S.A. 105, 6156-6161. doi: 10.1073/pnas.0711068105

Tanaka, H., Kitamura, M., Nakano, Y., Katayama, M., Takahashi, Y., Kondo, T., et al. (2012). CmpR is important for circadian phasing and cell growth. Plant Cell. Physiol. 53, 1561-1569. doi: 10.1093/pcp/pcs095

Toepel, J., Welsh, E., Summerfield, T. C., Pakrasi, H. B., and Sherman, L. A. (2008). Differential transcriptional analysis of the cyanobacterium Cyanothece sp. strain ATCC 51142 during light-dark and continuous-light growth. J. Bacteriol. 190, 3904-3913. doi: 10.1128/JB.00206-08

Vijayan, V., and O'Shea, E. K. (2013). Sequence determinants of circadian gene expression phase in cyanobacteria. J. Bacteriol. 195, 665-671. doi: 10.1128/JB.02012-12

Yen, U.-C., Huang, T.-C., and Yen, T.-C. (2004). Observation of the circadian photosynthetic rhythm in cyanobacteria with a dissolved-oxygen meter. Plant. Sci. 166, 949-952. doi: 10.1016/j.plantsci.2003.12.005

Conflict of Interest Statement: The authors declare that the research was conducted in the absence of any commercial or financial relationships that could be construed as a potential conflict of interest.

Received: 05 August 2013; accepted: 21 November 2013; published online: 06 December 2013.

Citation: Gaudana SB, Krishnakumar S, Alagesan S, Digmurti MG, Viswanathan GA, Chetty $M$ and Wangikar PP (2013) Rhythmic and sustained oscillations in metabolism and gene expression of Cyanothece sp. ATCC 51142 under constant light. Front. Microbiol. 4:374. doi: 10.3389/fmicb.2013.00374

This article was submitted to Microbial Physiology and Metabolism, a section of the journal Frontiers in Microbiology.

Copyright (c) 2013 Gaudana, Krishnakumar, Alagesan, Digmurti, Viswanathan, Chetty and Wangikar. This is an open-access article distributed under the terms of the Creative Commons Attribution License (CC BY). The use, distribution or reproduction in other forums is permitted, provided the original author(s) or licensor are credited and that the original publication in this journal is cited, in accordance with accepted academic practice. No use, distribution or reproduction is permitted which does not comply with these terms. 